\title{
FACE ANNOTATION FOR ONLINE PERSONAL VIDEOS USING COLOR FEATURE FUSION BASED FACE RECOGNITION
}

\author{
Jae Young Choi ${ }^{1}$, Konstantinos N. Plataniotis ${ }^{2}$, Yong Man Ro ${ }^{1}$, \\ ${ }^{1}$ Image and Video System Laboratory, Korea Advanced Institute of Science and Technology (KAIST), \\ Yuseong-Gu, Daejeon, 305-701, Republic of Korea, ${ }^{2}$ The Edward S Rogers Sr Department of Electrical \\ and Computer Engineering, University of Toronto, Toronto, Ontario, M5S 3GA, Canada \\ Email: jygchoi@kaist.ac.kr, kostas@comm.utoronto.ca, ymro@ee.kaist.ac.kr
}

\begin{abstract}
This paper proposes a novel weighted feature fusion in color face recognition (FR) to automatically annotate faces in personal videos. In the proposed FR method, multiple face images (belonging to the same subject) are clustered from a sequence of video frames. To facilitate a complementary effect on improving annotation performance, the grouped faces are combined using the proposed weighted feature fusion. In addition, we make effective use of facial color feature to cope with decrease in annotation performance due to a low-resolution face in personal videos. To evaluate the effectiveness of proposed FR method, more than 40,000 video frames for 10 real-world personal videos are collected from an existing online video sharing website. Experimental results show that the proposed FR method significantly improves annotation performance obtained using conventional grayscale image based FR methods.
\end{abstract}

Keywords - Face annotation, face recognition, personal videos, video annotation, color information

\section{INTRODUCTION}

Online video sharing websites, such as YouTube [1], have become indispensible resources for managing and sharing personal videos. As the amount of personal videos (on online video sharing websites) is explosively growing, automatic annotation of video [2] is of great importance for effective searching, browsing, and categorization. In particular, the act of labeling identities (i.e., the name of individuals) on personal videos, so-called face annotation (or tagging), is of considerable practical interest for video sharing websites due to high commercialization potential [3].

To realize an automatic face annotation system, computer-based face detection and face recognition (FR) techniques should be integrated. As reported in [4], the performance of face detection algorithms has improved considerably over the last decade. In contrast, traditional grayscale still-image based FR solutions [5] are still far from adequate for practical applications dealing with personal videos. This is mainly because the face images detected from personal videos are often subject to poor resolution and severe variations in illumination and viewpoint (see Fig. 2).

Recently, some research efforts have been made to take advantage of a temporal video sequence to overcome the limitations of traditional still-image based FR approaches [6-8]. In [6], to recognize faces in a test video sequence, a maximum a posteriori probability is computed by integrating the likelihood that each of the testing images belongs to a particular pose manifold. In [7], Multivariate Gaussian model is used to obtain the densities of multiple input images, acquired from a video sequence, and training images for each registered subject. The KL-divergence between the estimated model density and the input probability density is then computed in order to determine the true identity of an input image. Also the authors in [8] proposed the Mutual Subspace Method (MSM) in which similarity is defined by the angle between the input and the reference subspaces each constructed from a testing frame sequence and pre-enrolled images, respectively.

Previous works reviewed above have successfully demonstrated that temporal information is helpful for improving FR accuracy. However, when applying previous video-based FR methods to face annotation in personal videos, two major drawbacks can be identified: 1) Previous methods require a large corpus of realistic training data in order to reliably build probability models or subspaces for the purpose of using temporal information; acquiring a sufficient number of training samples may be difficult for FR applications in online video sharing websites as it requires an intensive manual labeling effort by human operator; 2) one crucial problem for recognizing faces on personal videos is to cope with low-resolution face images [2] (e.g., caused by using inexpensive webcams); however, this problem is little attention in previous works.

In this paper, we propose a novel weighted feature fusion based color FR for automated face annotation in personal videos. We aim at achieving a level of accuracy enough to be feasible in real-life applications and also low 
computational complexity for implementation. The main technical contributions of the proposed FR method are:

1) To take advantage of multiple face images (belonging to the same subject) that are able to be acquired from a sequence of video frames, we propose the effective weighted feature fusion for FR. This method has been designed to exploit a complementary effect by accounting for the confidence of each individual face feature (as obtained for each corresponding face image detected from a frame).

2) In [9], it has been found that facial color information can significantly improve the FR performance deteriorated by much lower resolution images. As such, we apply colorbased facial feature to the proposed weighted feature fusion FR to make an annotation system robust to lowresolution images.

3) To cope with a training-samples insufficiency problem, a generic-learning (GL) training scheme [10] is incorporated into the proposed FR method. Hence, face annotation accuracy of our method does not suffer from a shortage of training samples.

To assess the effectiveness of proposed FR method, more than 40,000 video frames from 10 real-world personal videos were collected from an existing online video sharing website. Experimental results show that the proposed method significantly improves the annotation accuracy, compared to the baseline FR in which only a single grayscale image is used for the purpose of FR.

The remainder of this paper is organized as follows. Section 2 presents an overview of the proposed face annotation framework. The face clustering method is briefly outlined in Section 3. Section 4 explains the proposed weighted feature fusion based color FR. Section 5 presents the experimental results to demonstrate the effectiveness of the proposed method. The conclusion is drawn in Section 6.

\section{OVERVIEW}

Fig. 1 provides an overview of the proposed face annotation framework. The proposed face annotation framework largely consists of three sequential steps: shot boundary detection, face clustering, and weighted feature fusion based color FR.

In general, shots are considered to be the primitives for video content based analysis, such as indexing and retrieval. In the proposed face annotation system, the shot boundary detection is performed using the method proposed in [11] in order to partition video sequence into a number of shots. The frames contained in each shot are applied to the face clustering step. The goal of face clustering is to group multiple face images (detected from a sequence of video frames within a shot) that belong to the same subject. Multiple face images contained in each face cluster are transformed into corresponding color face features. In sequence, multiple color face features of the same subject

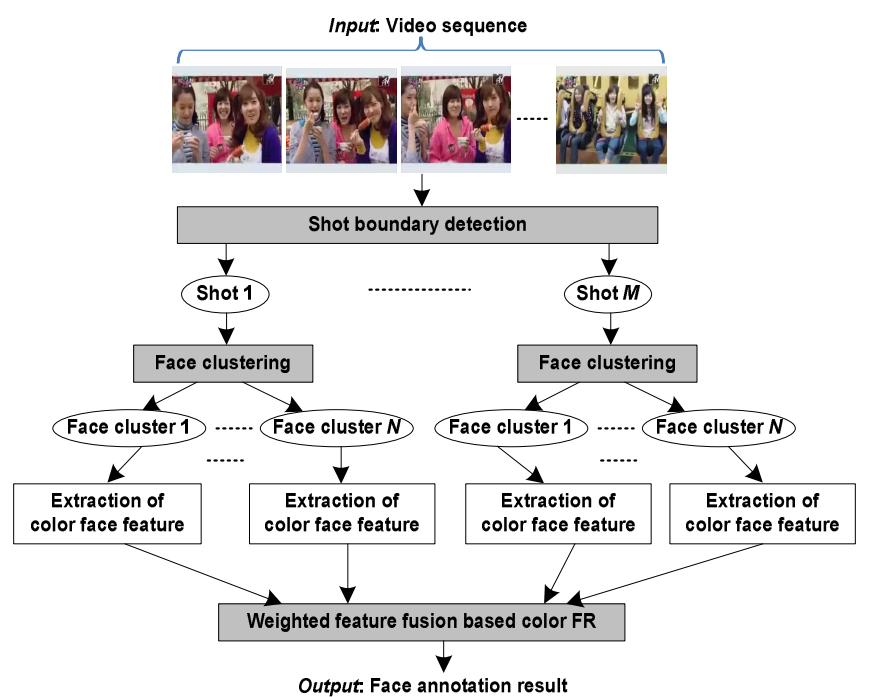

Fig. 1. Overview of the proposed face annotation framework.

are then combined using the proposed weighted feature fusion method. Finally, based on the obtained FR results, face images within a face cluster are annotated as the identity of a selected target subject. Face clustering and weighted feature fusion based color FR will be described in detail in the following sections.

\section{FACE CLUSTERING}

The ultimate goal of face clustering is twofold: first, all facial images of the same subject should be grouped in a single cluster and second, the face images of different subject should be part of different clusters.

We now explain our face clustering method. Let $\mathbf{I}_{i}$ be the $i^{\text {th }}$ face image detected from an associated video frame contained in a shot, where $1 \leq i \leq L$ and $L$ is the total number of face images that are detected from all of the video frames within a shot. Note that face images can be extracted using any state-of-the-art face detection technique [4]. Then we denote the face feature of $\mathbf{I}_{i}$ by $\mathbf{f}_{i}$. It should be noted that $\mathbf{f}_{i}$ can be obtained using any face feature extraction technique (e.g., using global or local face features [12]).

Since we have no a priori information about the possible identities of subjects or the nature of face feature samples, unsupervised clustering technique can be suitable for our face clustering purpose. For this reason, the averagelinkage based hierarchical agglomerative clustering (HAC) [19] is adopted as a face clustering method. The HAC-based face clustering algorithm is summarized below.

(a) For a single shot, assuming that a total of $L$ face features are available, HAC begins with $L$ singleton face clusters denoted by $\mathbf{C}_{i}$, where $i=1, \ldots, L$. Note that each $\mathbf{C}_{i}$ consists of a single face feature $\mathbf{f}_{i}$ at initial stage. 
(b) Calculate the average dissimilarity between $\mathbf{C}_{i}$ and $\mathbf{C}$, by summing the pairwise dissimilarities among the face features in two different face clusters as follows:

$$
D_{\text {cluster }}\left(\mathbf{C}_{i}, \mathbf{C}_{j}\right)=\frac{1}{\left|\mathbf{C}_{i}\right| \cdot\left|\mathbf{C}_{j}\right|} \cdot \sum_{\mathbf{f}_{m} \in \mathbf{C}_{i} i_{n} \in \mathbf{C}_{i}} D_{\text {face }}\left(\mathbf{f}_{m}, \mathbf{f}_{n}\right),
$$

where $|\cdot|$ denote the cardinality of a set, $D_{\text {face }}(\cdot)$ is a function that measure dissimilarity (e.g., distance) between a pair of face features, $m \neq n$, and $1 \leq m, n \leq L$.

(c) Find the nearest two face clusters $\mathbf{C}_{i}$ and $\mathbf{C}_{j}$ by comparing all $D_{\text {cluster }}\left(\mathbf{C}_{i}, \mathbf{C}_{j}\right)$ one by one in the following way:

$$
\left(\mathbf{C}_{i}, \mathbf{C}_{j}\right)=\arg \min _{i, j} D_{\text {cluster }}\left(\mathbf{C}_{i}, \mathbf{C}_{j}\right), \quad i \neq j .
$$

(d) Merge the two nearest clusters into a single cluster as being $\mathbf{C}_{i}=\mathbf{C}_{\hat{i}} \cup \mathbf{C}_{\hat{j}}$ and removing $\mathbf{C}_{\hat{i}}$ and $\mathbf{C}_{\hat{j}}$.

(d) If $D_{\text {cluster }}\left(\mathbf{C}_{i}, \mathbf{C}_{j}\right)$ were less than a prespecified stopping threshold $\xi$, then repeat (b), (c), and (d), else terminate the HAC clustering process.

In order to determine an optimal $\xi$, the stopping threshold selection technique proposed in [13] has been used in our HAC-based face clustering.

\section{WEIGHTED FEATUER FUSION BASED COLOR FACE RECOGNITION}

\subsection{Extracting color face feature}

For the sake of simplicity, we denote a certain face cluster by $\mathbf{C}$ without any loss of generality. Let $\mathbf{I}_{\mathrm{q}}^{(m)}$ and $\mathbf{I}_{\mathrm{t}}^{(n)}$ be the $m^{\text {th }}$ query face image (to be recognized) in the set of all face images within $\mathbf{C}$ (i.e., $\mathbf{I}_{q}^{(m)} \in \mathbf{C}$ ) and the $n^{\text {th }}$ target face image, respectively, where $1 \leq m \leq \mathbf{C} \mid, 1 \leq n \leq G$, and $G$ is the number of all of the target subjects that are enrolled in a target database. Note that $\mathbf{I}_{\mathrm{q}}^{(m)}$ and $\mathbf{I}_{\mathrm{t}}^{(n)}$ are assumed to be red-green-blue $(R G B)$ color face images.

In order to extract the color face features of $\mathbf{I}_{\mathrm{q}}^{(m)}$ and $\mathbf{I}_{\mathrm{t}}^{(n)}, \quad \mathbf{I}_{\mathrm{q}}^{(m)}$ and $\mathbf{I}_{\mathrm{t}}^{(n)}$ are first converted into a number of different color spaces. Let us assume that a total of $K$ different color component images are generated (e.g., luminance $(Y)$ or chrominance component $\left(C_{b}\right.$ or $\left.C_{r}\right)$ images in $Y C_{b} C_{r}$ color space). We denote the $k^{\text {th }}$ color component vectors [9] of $\mathbf{I}_{\mathrm{q}}^{(m)}$ and $\mathbf{I}_{\mathrm{t}}^{(n)}$ by $\mathbf{s}_{\mathrm{q}}^{(m, k)}$ and $\mathbf{s}_{\mathrm{t}}^{(n, k)}$, respectively, where $k=1, \ldots, K$. Note that each color component vector is a column vector by lexicographic ordering of the pixel elements of a 2-dimensional color component image.
Let us denote the $k^{\text {th }}$ feature extractor [5] by $\varphi_{k}$ that is formed with a training set consisting of the $k^{\text {th }}$ color component vectors. It is important to note that each $\varphi_{k}$ is assumed to be created with a GL-based training scheme (refer to [10] for further details). In GL-based training, when learning $\varphi_{k}$, a generic database comprising of subjects other than those to be recognized is used. Then the low-dimensional features of the $k^{\text {th }}$ color component vectors $\mathbf{s}_{\mathrm{q}}^{(m, k)}$ and $\mathbf{s}_{\mathrm{t}}^{(n, k)}$ are obtained using corresponding $\varphi_{k}$ as follows:

$$
\mathbf{f}_{\mathrm{q}}^{(m, k)}=\varphi_{k}\left(\mathbf{s}_{\mathrm{q}}^{(m, k)}\right) \text { and } \mathbf{f}_{\mathrm{t}}^{(n, k)}=\varphi_{k}\left(\mathbf{s}_{\mathrm{t}}^{(n, k)}\right), k=1, \ldots, K,
$$

where $\mathbf{f}_{\mathrm{q}}^{(m, k)}, \mathbf{f}_{\mathrm{t}}^{(n, k)} \in \mathbf{R}^{F_{k}}$ and $F_{k}$ denote dimensionality of both $\mathbf{f}_{\mathrm{q}}^{(m, k)}$ and $\mathbf{f}_{\mathrm{t}}^{(n, k)}$.

In order to generate the color face features of $\mathbf{I}_{q}^{(m)}$ and $\mathbf{I}_{t}^{(n)}, K$ complementary color component features are combined at the feature-level by concatenating them in column order:

$$
\begin{aligned}
& \mathbf{f}_{\mathrm{q}}^{(m)}=\left[\begin{array}{llll}
\left(\mathbf{f}_{\mathrm{q}}^{(m, 1)}\right)^{\mathrm{T}} & \left(\mathbf{f}_{\mathrm{q}}^{(m, 2)}\right)^{\mathrm{T}} & \ldots & \left(\mathbf{f}_{\mathrm{q}}^{(m, K)}\right)^{\mathrm{T}}
\end{array}\right]^{\mathrm{T}} \text { and } \\
& \mathbf{f}_{\mathrm{t}}^{(n)}=\left[\begin{array}{llll}
\left.\mathbf{f}_{\mathrm{t}}^{(n, 1)}\right)^{\mathrm{T}} & \left(\mathbf{f}_{\mathrm{t}}^{(n, 2)}\right)^{\mathrm{T}} & \cdots & \left(\mathbf{f}_{\mathrm{t}}^{(n, K)}\right)^{\mathrm{T}}
\end{array}\right]^{\mathrm{T}}
\end{aligned}
$$

where $\mathrm{T}$ denotes the transpose operator of the matrix, $\mathbf{f}_{\mathrm{q}}^{(m)}, \mathbf{f}_{\mathrm{t}}^{(n)} \in \mathbf{R}^{F}$, and $F=\sum_{k=1}^{K} F_{k}$. Note that $\mathbf{f}_{\mathrm{q}}^{(m, k)}$ and $\mathbf{f}_{\mathrm{t}}^{(n, k)}$ should be individually normalized to have zero mean and unit variance prior to their concatenation.

Subsequently, $\mathbf{f}_{q}^{(m)}(m=1, \ldots,|\mathbf{C}|)$ and $\mathbf{f}_{\mathrm{t}}^{(n)}(n=1, \ldots, G)$ are applied to FR step using weighted feature fusion in order to annotate face images $\left(\mathbf{I}_{\mathrm{q}}^{(m)}\right)$ available in $\mathbf{C}$. This process will be discussed in more detail in the following subsection.

\subsection{Face recognition relying on weighted feature fusion}

This section explains an FR method using a weighted feature fusion of multiple color face features. Note that several defective face images arising from variations in appearance including viewpoints and illumination may be included in a face cluster. We regard such defective face images as outliers comparing to a prototype (or representative) image for a single face cluster. The outliers presented in a face cluster should be handled by properly reducing their impacts before consolidating them. To this end, we impose a weight on each individual $\mathbf{f}_{q}^{(m)}$ underpinning the distance from a corresponding prototype.

It is well-known that the median is more resilient to outliers than the mean. Hence, we adopt a median feature vector, denoted by $\widetilde{\mathbf{f}}_{q}$, as a prototype for a face cluster. Note that each element of $\widetilde{\mathbf{f}}_{q}$ is filled with the median value 
of all corresponding elements of $\mathbf{f}_{\mathrm{q}}^{(m)}(m=1, \ldots,|\mathbf{C}|)$. To diminish the influence of the elements of $\mathbf{f}_{\mathrm{q}}^{(m)}$ that are far away from the $\widetilde{\mathbf{f}}_{q}$, a penalty based Minkowski distance is defined as follows:

$$
d_{m}=\left(\sum_{k}\left\{\Omega\left(\left|\Gamma_{k}\left(\tilde{\mathbf{f}}_{\mathrm{q}}\right)-\Gamma_{k}\left(\mathbf{f}_{\mathrm{q}}^{(m)}\right)\right|\right)\right\}^{p}\right)^{\frac{1}{p}},
$$

where

$$
\Omega(|x|)= \begin{cases}\tau \cdot|x| & \text { if }|x|>\delta \cdot \sigma_{k} \\ |x| & \text { otherwise }\end{cases}
$$

and $\Gamma_{k}(\cdot)$ is a function that returns the $k^{\text {th }}$ element of the argument vector, $\sigma_{k}$ stands for the standard deviation computed over the $k^{\text {th }}$ element samples of the feature vectors $\mathbf{f}_{\mathrm{q}}^{(m)}$ that are part of $\mathbf{C}$, and $\delta$ and $\tau$ denotes a user-specific threshold and a penalty constants, respectively. Based on our experimentation, 2.2 and 2.0 are found to be reasonable values for $\delta$ and $\tau$, respectively. It should be emphasized that in (5), the distance $d_{m}$ is forced to be increased if the difference between each element in $\widetilde{f}_{q}$ and that in $\mathbf{f}_{\mathrm{q}}^{(m)}$ exceeds a certain $\delta \cdot \sigma_{k}$. The actual increase is controlled by the parameter $\tau$ (see (6)).

Using $d_{m}$ given by (5) and a soft-max function [14], we compute the weight in order to adjust the influence of $\mathbf{f}_{\mathrm{q}}^{(m)}$ on the fusion of face features:

$$
w_{m}=\frac{\exp \left(-d_{m}\right)}{\sum_{m=1}^{|\mathbf{C}|} \exp \left(-d_{m}\right)} .
$$

Note that $d_{m}$ should be normalized to have zero mean and unit standard deviation prior to the computation of $w_{m}$. In this paper, the widely used 'z-score' technique is employed to normalize the distance scores. Other distance score normalization techniques are explained in detail in [15].

Using $w_{m}$, a single feature vector can be computed as a weighted average of the individual feature vectors $\mathbf{f}_{\mathrm{q}}^{(m)}$ in C as follows:

$$
\mathbf{f}_{\mathrm{q}}=\sum_{m=1}^{|\mathrm{C}|} w_{m} \cdot \mathbf{f}_{\mathrm{q}}^{(m)} .
$$

In (8), by assigning a higher weight to the reliable face features and a lower weight to the other face features (i.e., the outliers), the chance of assigning such outliers to the wrong subject class can be reduced.

To perform the face annotation on $\mathbf{I}_{\mathrm{q}}^{(m)}$, a nearest neighbor classifier is then applied to determine the identity of $\mathbf{I}_{\mathrm{q}}^{(m)}$ by finding the smallest distance between $\mathbf{f}_{\mathrm{q}}^{(m)}$ and $\mathbf{f}_{\mathrm{t}}^{(n)}(n=1, \ldots, G)$, as follows:

$$
l\left(\mathbf{I}_{\mathrm{q}}^{(m)}\right)=l\left(\mathbf{I}_{\mathrm{t}}^{\left(n^{*}\right)}\right) \text { and } n^{*}=\underset{n=1}{G} D_{\text {face }}\left(\mathbf{f}_{\mathrm{q}}, \mathbf{f}_{\mathrm{t}}^{(n)}\right),
$$

where $l(\cdot)$ is a function that returns the identity of an input face image. Using (9), all $\mathbf{I}_{\mathrm{q}}^{(m)}$ contained in $\mathbf{C}$ are annotated as subject identity $l\left(\mathbf{I}_{t}^{\left(n^{*}\right)}\right)$ in a batch manner.

\section{EXPERIMENTS}

To demonstrate the effectiveness of the proposed method, 10 real-world personal videos were collected from the 'YouTube' [1] video sharing website. More than 40,000 video frames of collected personal videos were then utilized. They include real events, such as birthday party, wedding, gathering, and trip, etc. To form a corresponding groundtruth face image set for each personal video, Viola Jone's face detection [16] was first ran on all video frames and then the identity of each detected face image was manually labeled. Note that, in order to focus on investigating the face annotation accuracy related to FR, we excluded face detection error rates from our performance evaluation. As a result, we constructed the 10 ground truth sets, each of which consists of average 1,996 facial images for average 20 subjects.

Fig. 2 shows examples of the video frame sequence as well as the detected face images that are part of the ground truth. As shown in Fig. 2, detected face images are significantly challenging for FR due to very low-resolution, severe pose variation, and even occlusion.

In the face clustering step, popular Local Binary Pattern (LBP) feature [18] was adopted as a face feature. In addition, the three popular face feature extraction algorithms were used to form the face feature extractor for the purpose of computing color face feature described in (3): Principal Component Analysis (PCA), Fisher's Linear Discriminant Analysis (FLDA), and Bayesian [17]. In addition, to perform the GL-based training during construction of a face feature extractor, a generic training set of $4,474 R G B$ color facial images of 887 subjects were collected from CMU and Color FERET public databases.

In this experiment, we evaluate the performance of the proposed face clustering method discussed in Section 3. It should be noted that the final goal of shot boundary detection and face clustering is to group face images belonging to the same subject as correctly as possible. For this reason, this experiment focuses on assessing the accuracy of merging face images by using both shot boundary detection and face clustering rather than assessing the accuracy of shot boundary detection alone. In order to consider the aforementioned issues during the evaluation of the face clustering performance, the FScore metric [19] was 


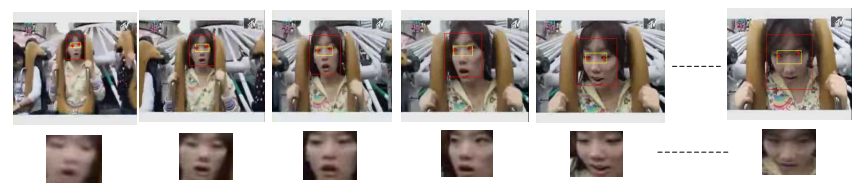

Fig. 2. Examples of video frame sequence and face images that are part of the ground truth. The detected face images are placed to the bottom side of each corresponding video frame. Note that all of the detected face images were individually rotated and rescaled to $86 \mathrm{x}$ 86 pixels so that eye centers were placed on known pixel locations (as recommended by the FERET protocol [17]).

Table 1. FScore values averaged over all of the video shots generated for each personal video. Note that FScore value is ranging from 0 to 1 .

\begin{tabular}{ccc}
\hline \hline $\begin{array}{c}\text { Name of personal } \\
\text { video }\end{array}$ & Average FScore & $\begin{array}{c}\text { Average optimal } \\
\text { stopping threshold }\end{array}$ \\
\hline \hline Video 1 & 0.87 & 0.41 \\
\hline Video 2 & 0.77 & 0.52 \\
\hline Video 3 & 0.86 & 0.56 \\
\hline Video 4 & 0.92 & 0.63 \\
\hline Video 5 & 0.74 & 0.49 \\
\hline Video 6 & 0.88 & 0.51 \\
\hline Video 7 & 0.92 & 0.66 \\
\hline Video 8 & 0.87 & 0.58 \\
\hline Video 9 & 0.85 & 0.59 \\
\hline Video 10 & 0.91 & 0.64 \\
\hline
\end{tabular}

adopted to quantify the clustering performance. As described in [19], FScore value is ranging from 0 to 1 . This indicates that FScore will be one in the case where every subject has a corresponding face cluster that contains all face images belonging to a unique subject. Hence, the higher the FScore value, the better the clustering result in the sense of natural grouping. In addition, for a face annotation performance metric, the $H$-Hit rate [20] was used. If the true name of a given query face is actually in the list containing $H$ names, this query face is said to be 'hit' by the name list.

In order to investigate face clustering performance, the resulting FScores for each personal video used are shown in Table 1. It is important to note that all FScores shown in Table 1 are values averaged over all video shots produced for each personal video. In addition, average FScores are computed at corresponding average optimal stopping threshold (determined using the method proposed in [13]). Looking into the results in Table 1, FScores achieve more than 0.85 for most of the personal videos used. Based on the fact that the FScore becomes one when perfect clustering results are achieved, we can demonstrate that the proposed face clustering method are able to attain a reliable clustering performance (for an optimal stopping threshold).

Fig. 3 shows the comparisons of the $H$-Hit rates of the proposed FR method with the $H$-Hit rates of the baseline FR method. It should be noted that all results presented in Fig. 3 are averaged over the 10 different personal videos. In Fig. 3, the performance of conventional appearance-based FR solutions [5] (only using a single grayscale face image) is referred to as baseline face annotation accuracy. Baseline
FR

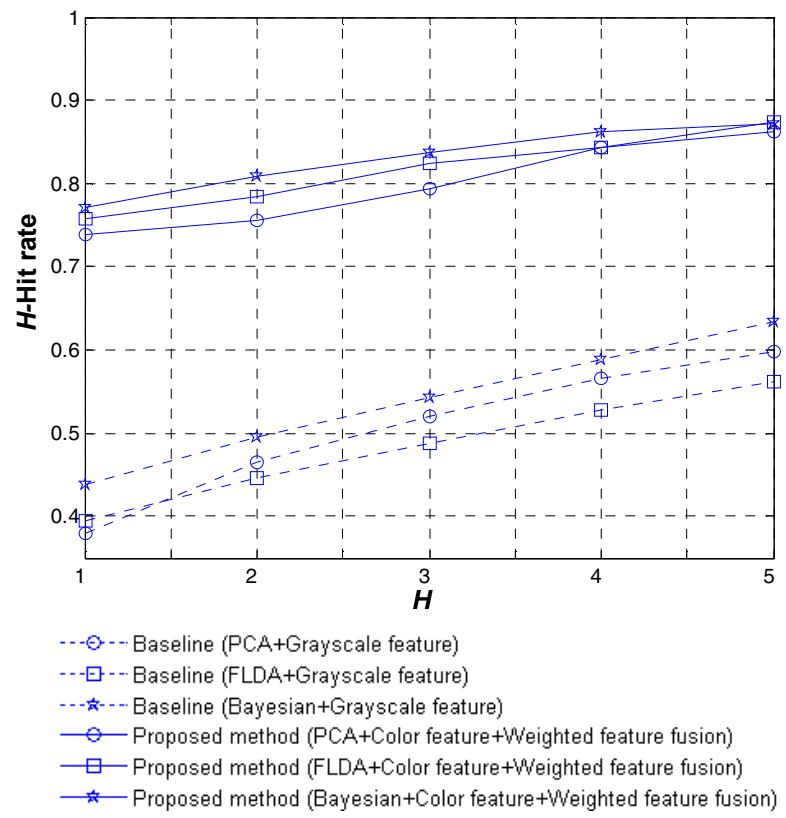

Fig. 3. Comparisons of the $H$-Hit rates obtained for the baseline FR method and the proposed FR method using three different feature extraction algorithms. Note that ' $R$ ' [9] from ' $R G B$ ' color space was used for a grayscale face feature in Baseline FR, while ' $R Q C r$ ' [9] color component images ( $Q$ and $C_{r}$ from $Y I Q$ and $Y C_{b} C_{r}$ color spaces) were used to form a color face feature.

also utilizes a training set that consists of training images corresponding to the target subjects. It should be noted that, when referring to the literature in the area of FR [10], [12], the use of eight training images is usually sufficient to prevent a significant decrease in the FR performance caused by a shortage of training images. For this reason, the training set for baseline FR contained eight face images per target subject in our experiments. This guarantees fair and stable comparisons with the proposed FR method that relies on a GL-based training scheme (requiring no training face images) and weighted face feature fusion.

In Fig. 3, we can see that the face annotation task for personal videos collected from 'YouTube' is significantly challenging. Specifically, the average $H$-Hit rates obtained for baseline FR are noticeably low (less than 45 percent) for all feature extraction methods used. However, we can see that a substantial improvement in annotation performance can be achieved by the proposed FR method, although the face clustering error is included as described in Table 1. In particular, the 1 -Hit rate, averaged over 10 personal videos, can be improved with around $35.89 \%, 36.28 \%$, and $33.30 \%$, for PCA, FLDA, and Bayesian, respectively.

Fig. 4 demonstrates the usefulness of color face feature for enhancing face annotation accuracy of the proposed weighted feature fusion based FR method. The 1-Hit rates are averaged over the 10 personal videos, and the grayscale 
and the color face features are the same as those used to

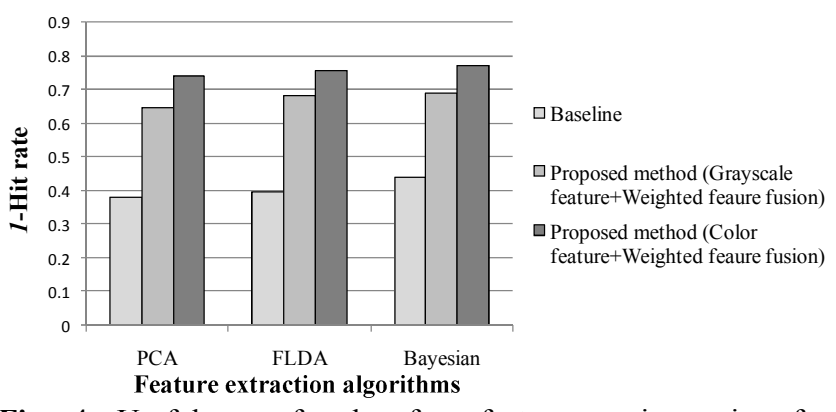

Fig. 4. Usefulness of color face feature on improving face annotation accuracy of the proposed weighted feature fusion based FR.

baseline FR are presented for comparison purpose. As shown in Fig. 4, further improvement in annotation accuracy can be observed by making use of color face feature in comparison to the case where grayscale feature is only used. This result indicates that facial color information is useful for improving annotation accuracy of face annotation system targeting to personal videos, which frequently have to cope with much low-resolution face images.

\section{CONCLUSIONS}

In this paper, we demonstrate that the substantial improvement in annotating faces on personal videos can be achieved by combining multiple face images in way of weighted feature fusion. In addition, it is found that facial color information can be useful to cope with much lower face resolution problem, which is critical in most videobased FR applications. Considering the reliable face annotation accuracy and the low system complexity, we believe that the proposed face annotation method would be readily applicable to the promising subject-based personal video indexing and retrieval applications.

For the future work, we will incorporate face tracking technique [5] into face clustering step in order to more correctly group multiple face images that belong to the same subject.

\section{REFERENCES}

[1] [Online]. Available: http://www.youtube.com.

[2] N. Morsillo, G. Mann, and C. Pal, "YouTube Scale, Large Vocabulary Video Annotation," in Video Search and Minining. New-York: Springer-Verlag, 2008.

[3] A. Matellanes, A. Evans, and B. Erdal, "Creating an application for automatic annotation of images and video," Proc. of IEEE Int'l Conf. on SWAMM, 2007.

[4] M. H. Yang, D. J. Kriegman, and N. Ahuja, "Detecting Faces in Images: A Survey," IEEE Trans, Pattern. Anal. Machine. Intell, vol. 24, no. 1, pp. 34-58, 2002.

[5] W. Zhao, R. Chellappa, P. J. Phillips, and A. Rosenfeld, "Face Recognition: A Literature Survey," ACM Comput. Surv., vol. 35, no. 4, pp. 399-458, 2003. obtain the results depicted in Fig. 3. Also 1-Hit rates of the

[6] K. C. Lee, J. Ho, M. H. Yang, and D. Kriegman, "VideoBased Face Recognition Using Probabilistic Appearance Manifolds", Proc. of IEEE Int'l Conf. on CVPR, 2003.

[7] G. Shakhnarovich, J. W. Fisher, and T. Darrell, "Face recognition from long-term observations", Proc. of European Conf. on Computer Vision, 2002.

[8] O. Yamaguchi, K. Fukui, and K. Maeda, "Face recognition using temporal image sequence", Proc. of IEEE Int'l Conf. on $A F G R, 1998$.

[9] J. Y. Choi, Y. M. Ro, and K. N. Plataniotis, "Color Face Recognition for Degraded Face Images," IEEE Trans. Systems. Man and Cybernetics-Part B, vol. 39, no. 5, pp. 1217-1230, 2009.

[10] J. Wang, K. N. Plataniotis, J. Lu, and A.N. Venetsanopoulos, "On solving the face recognition problem with one training sample per subject," Pattern Recognit., vol. 39, no. 6, pp. 1746-1762, 2006.

[11] S.H. Shim and Yong Man Ro, "Real-time shot boundary detection for digital video camera using the MPEG-7 descriptor," SPIE Int'l Conf. on Electronic Imaging, 2002.

[12] Y. Su, S. Shan, X. Chen, and W. Gao, "Hierarchical Ensemble of Global and Local Classifiers for Face Recognition," IEEE Trans. Image Processing, vol. 18, no. 8, pp. 1885-1886, 2009.

[13] Y.J Jung, H.S Park, D.Z. Du, and B. L. Drake, "A Decision Criterion for the Optimal Number of Clusters in Hierarchical Clustering," Journal of Global Optimization, vol. 25, pp. 91111,2003

[14] C. M. Bishop, Pattern Recognition and Machine Learning. New York: Springer-Verlag, 2006.

[15] A. Jain, K. Nandakumar, and A. Ross, "Score normalization in multimodal biometric systems," Pattern Recognit., vol. 38, no. 12, pp. 2270-2285, 2005.

[16] P. Viola and M. Jones, "Rapid Object Detection using a Boosted Cascade of Simple Features," Proc. IEEE Int'l Conf. on CIVR, 2001.

[17] P. J. Phillips, H. Moon, S. A. Rizvi, and P. J. Rauss, "The FERET Evaluation Methodology for Face Recognition Algorithms," IEEE Trans. Pattern Anal. Mach. Intell., vol. 22, no. 10, pp. 1090-1104, 2000.

[18] T. Ahonen, A. Hadid, and M. Pietikainen, "Face Description with Local Binary Pattern: Application to Face Recognition," "IEEE Trans. Pattern Anal. Mach. Intell., vol. 28, no. 12, pp. 2037-2041, 2006.

[19] Y. Zhao and G. Karypis, "Hierarchical Clustering Algorithms for Document Datasets," Data Mining and Knowledge Discovery, vol. 10, pp. 141-168, 2005.

[20] L. Chen, B. Hu, L. Zhang, M. Li, and H.J. Zhang, "Face annotation for family photo album management," International Journal of Image and Graphics, vol. 3, no. 1, pp. 1-14, 2003. 